Volume 11, Issue 12, December 2020, pp. 2147-2157. Article ID: IJM_11_12_202

Available online at http://iaeme.com/Home/issue/IJM?Volume $=11 \&$ Issue $=12$

Journal Impact Factor (2020): 10.1471 (Calculated by GISI) www.jifactor.com

ISSN Print: 0976-6502 and ISSN Online: 0976-6510

DOI: 10.34218/IJM.11.12.2020.202

\title{
MODEL FOR IDENTIFYING VOLATILITY IN \\ FINANCIAL MARKETS WITH THE HELP OF NSE LISTED STOCKS WITH SPECIAL REFERENCE TO AXIS BANK LIMITED, BAJAJ FIN SERVE LIMITED AND BAJAJ AUTO LIMITED DURING POST COVID-19 IN INDIA- AN EXPLORATORY STUDY
}

\author{
R. Amuthan \\ Associate Professor, Department of Management Studies, \\ Karunya (Deemed) University, Coimbatore, Tamilnadu, India \\ V. Lawrance \\ Professor, Department of Management Studies, \\ Karunya(Deemed) University, Coimbatore, Tamilnadu, India \\ Dr. K. Kumara Pillai \\ Professor, Department of Management Studies, \\ Karunya(Deemed) University, Coimbatore, Tamilnadu, India
}

\begin{abstract}
This study covers the whole gamut of volatility in terms of Share price increase or decrease. The centre of coverage over here in this study is COVID-19. We all have to strongly admit that COVID has created much more crucified impacts on all businesses including stock market trading. 100 Trading Days Cash Market data and 90 trading days Future Price data were taken in to account. Volatility is being computed with the help of Excel application and it is found that those stocks experienced low upswings are said to be high risky stocks and these stocks are not fit enough to buy. These share prices are further backed up by Cash flows, Book Value Per share, Earnings Per share. These financial variables' quarterly growth history from the start of Lock down is checked out and if the growth is negative, the concerned stock is classified as risk and it is not fit to buy.
\end{abstract}


Model for Identifying Volatility in Financial Markets with the help of NSE Listed Stocks with special reference to Axis Bank Limited, Bajaj Fin serve Limited and Bajaj Auto Limited during Post COVID19 in India-An Exploratory Study

Key words: Volatility, Upswing, Downswing, Fit to Buy for Investment and Trading

Cite this Article: R. Amuthan, V. Lawrance and Dr. K. Kumara Pillai, Model for Identifying Volatility in Financial Markets with the help of NSE Listed Stocks with special reference to Axis Bank Limited, Bajaj Fin serve Limited and Bajaj Auto Limited during Post COVID-19 in India-An Exploratory Study, International Journal of Management, 11(12), 2020, pp 2147-2157.

http://iaeme.com/Home/issue/IJM?Volume=11\&Issue=12

\section{INTRODUCTION}

It is a rate at which the price of a security increases or decreases for a given set of returns. Volatility is measured by calculating the standard deviation of the annualized returns over a given period of time. It shows the range to which the price of a security may increase or decrease. Volatility measures the risk of a security. It is used in option pricing formula to gauge the fluctuations in the returns of the underlying assets. Volatility indicates the pricing behavior of the security and helps estimate the fluctuations that may happen in a short period of time. If the prices of a security fluctuate rapidly in a short time span, it is termed to have high volatility. If the prices of a security fluctuate slowly in a longer time span, it is termed to have low volatility.

\subsection{Statement of Problem}

In Equity Market, detecting the real stocks which are yielding returns is found to be difficult on account of very high volatility in the Market. Hence there is the strong and reliable analysis are required based on certain criteria.

\subsection{Objectives of the Study}

To describe the model to find out Highly Volatile and Less Volatile Stocks

\subsection{Need for the Study}

- To inform novice investors about the whole landscape of stock market fluctuations

- To tell them how to make money out of stock market investments

\subsection{Scope of Study}

This study explains the methodology of finding stock market volatility with the evidence of Apollo Tyres Limited, Asian Paints Limited and Ashok Leyland Limited. 101 trading days in Cash Market and 90 Trading Days in Derivative Market were taken to prove the volatility impacts.

\subsection{Limitations}

This study derives its findings and conclusions only by means of analyzing Financial Data. All drawbacks using financial data will be reflected in this study also.

\section{FINANCIAL RATIOS}

\subsection{Return on Capital Employed (ROCE)}

ROCE is a profitability ratio which measures how efficiently a company can generate profits from the capital employed. The return on capital employed shows how much operating income is generated for each rupee of capital invested. A high and stable ROCE can be a sign of a very good company, as it shows that a firm is making consistently good use of its resources. 
However, a higher ROCE is more favorable always, as it indicates that more profits are generated for each rupee of capital employed.

The formula is given by,

$$
\text { ROCE }=\frac{E B I T}{\text { Capital Employed }}
$$

where Capital Employed $=$ Total Assets - Current Liabilities

Table 1 Total Assets (in crores)

\begin{tabular}{|l|l|l|l|l|}
\hline \multicolumn{1}{|c|}{ Company } & \multicolumn{1}{c|}{$\mathbf{2 0 1 9}$} & \multicolumn{1}{c|}{$\mathbf{2 0 1 8}$} & \multicolumn{1}{c|}{$\mathbf{2 0 1 7}$} & \multicolumn{1}{c|}{$\mathbf{2 0 1 6}$} \\
\hline Axis Bank & $8,009,965,316$ & $6,913,295,799$ & $6,014,676,703$ & $5,254,676,181$ \\
\hline Bajaj Finserve Ltd. & $2,08,548.71$ & $1,59,126.14$ & $1,28,617.27$ & $1,03,445.03$ \\
\hline Bajaj Auto Ltd. & $28,834.41$ & $25,141.00$ & $21,637.62$ & $16,426.63$ \\
\hline
\end{tabular}

Table 2 Current Liabilities (in crores)

\begin{tabular}{|l|l|l|l|l|}
\hline \multicolumn{1}{|c|}{ Company } & \multicolumn{1}{c|}{$\mathbf{2 0 1 9}$} & \multicolumn{1}{c|}{$\mathbf{2 0 1 8}$} & \multicolumn{1}{c|}{$\mathbf{2 0 1 7}$} & \multicolumn{1}{c|}{$\mathbf{2 0 1 6}$} \\
\hline Axis Bank & $330,731,159$ & $262,454,534$ & $262,954,713$ & $151,087,716$ \\
\hline Bajaj Finserve Ltd. & $61,263.28$ & $56,738.85$ & $50,389.78$ & $38,102.93$ \\
\hline Bajaj Auto Ltd. & $4,873.78$ & $4,111.40$ & $3,212.84$ & $2,953.09$ \\
\hline
\end{tabular}

Table 3 Capital Employed (in crores)

\begin{tabular}{|l|l|l|l|l|}
\hline \multicolumn{1}{|c|}{ Company } & \multicolumn{1}{|c|}{$\mathbf{2 0 1 9}$} & \multicolumn{1}{c|}{$\mathbf{2 0 1 8}$} & \multicolumn{1}{c|}{$\mathbf{2 0 1 7}$} & \multicolumn{1}{c|}{$\mathbf{2 0 1 6}$} \\
\hline Axis Bank & $7,679,234,157$ & $6,650,841,265$ & $5,751,721,990$ & $5,103,588,465$ \\
\hline Bajaj Finserve Ltd. & 147,285 & 102,387 & 78,227 & 65,342 \\
\hline Bajaj Auto Ltd. & 23,961 & 21,030 & 18,425 & 13,474 \\
\hline
\end{tabular}

Table 4 EBIT (in crores)

\begin{tabular}{|l|l|l|l|l|}
\hline \multicolumn{1}{|c|}{ Company } & \multicolumn{1}{c|}{$\mathbf{2 0 1 9}$} & \multicolumn{1}{c|}{$\mathbf{2 0 1 8}$} & \multicolumn{1}{c|}{$\mathbf{2 0 1 7}$} & \multicolumn{1}{c|}{$\mathbf{2 0 1 6}$} \\
\hline Axis Bank & $69,740,881$ & $1,215,715$ & $54,675,647$ & $126,820,480$ \\
\hline Bajaj Finserve Ltd. & $8,154.74$ & $6,310.17$ & $4,924.53$ & $3,804.05$ \\
\hline Bajaj Auto Ltd. & $6,955.58$ & $5,933.41$ & $5,587.55$ & $5,295.31$ \\
\hline
\end{tabular}

Table 5 Return on Capital Employed (ROCE)

\begin{tabular}{|l|l|l|l|l|}
\hline \multicolumn{1}{|c|}{ Company } & \multicolumn{1}{|c|}{$\mathbf{2 0 1 9}$} & \multicolumn{1}{|c|}{$\mathbf{2 0 1 8}$} & \multicolumn{1}{|c|}{$\mathbf{2 0 1 7}$} & \multicolumn{1}{|c|}{$\mathbf{1 0 1 6}$} \\
\hline Axis Bank & 0.91 & 0.02 & 0.95 & 2.48 \\
\hline Bajaj Finserve Ltd. & 5.54 & 6.16 & 6.30 & 5.82 \\
\hline Bajaj Auto Ltd. & 29.03 & 28.21 & 30.33 & 39.30 \\
\hline
\end{tabular}

ROCE of Axis Bank and Bajaj Auto has a steady decline between 2016 and 2018. Whereas, in 2019 it has increased. Bajaj Finserve's ROCE has a rise and fall between 2016 and 2018.

\subsection{Cash Flow from Operations (CFO)}

It represents the amount of cash a company generates (or consumes) from carrying out its operating activities over a period of time.

Operating cash flow is calculated by starting with net income. The income statement includes expenses that may not have actually been paid for yet. Thus, net income has to be adjusted by adding back all non-cash expenses like depreciation, stock-based compensation, and others.

The following table shows the cash flow from operating activities (in crores) taken from the financial statements of respective companies. 
Model for Identifying Volatility in Financial Markets with the help of NSE Listed Stocks with special reference to Axis Bank Limited, Bajaj Fin serve Limited and Bajaj Auto Limited during Post COVID19 in India-An Exploratory Study

Table 6 Cash Flow from Operations (in crores)

\begin{tabular}{|l|l|l|l|l|}
\hline \multicolumn{1}{|c|}{ Company } & \multicolumn{1}{|c|}{$\mathbf{2 0 1 9}$} & \multicolumn{1}{c|}{$\mathbf{2 0 1 8}$} & \multicolumn{1}{c|}{$\mathbf{2 0 1 7}$} & \multicolumn{1}{c|}{$\mathbf{2 0 1 6}$} \\
\hline Axis Bank & $37,125.25$ & $-38,390.30$ & $34,192.28$ & $-31,076.68$ \\
\hline Bajaj Finserve Ltd. & $-27,102.22$ & $-2,037.69$ & $-1,218.88$ & -583.96 \\
\hline Bajaj Auto Ltd. & $2,486.86$ & $4,327.84$ & $3,267.36$ & $3,678.45$ \\
\hline
\end{tabular}

Bajaj Auto has positive cash flow, whereas Bajaj Finserve has negative cash flow. Axis bank's CFO is fluctuating.

\subsection{Book Value Per Share (BVPS)}

It is the per share value of a company based on its equity available to common shareholders. The book value is a company's assets minus its liabilities. It may also be called as shareholder's equity.

\section{Shareholder's Equity}

$\mathrm{BV} \mathrm{PS}=$

\section{Outstanding Shares}

Book value per share is highly useful for investors to get a real-world view of a company's equity value. It may be used by some investors to determine the equity in a company relative to the market value of the company, which is the price of its stock.

Table 7 Book Value Per Share (Rs.)

\begin{tabular}{|l|l|l|l|l|}
\hline \multicolumn{1}{|c|}{ Company } & \multicolumn{1}{c|}{$\mathbf{2 0 1 9}$} & \multicolumn{1}{c|}{$\mathbf{2 0 1 8}$} & \multicolumn{1}{c|}{$\mathbf{2 0 1 7}$} & \multicolumn{1}{c|}{$\mathbf{2 0 1 6}$} \\
\hline Axis Bank & 263.65 & 250.17 & 235.41 & 224.77 \\
\hline Bajaj Finserve Ltd. & $2,296.58$ & $1,964.14$ & $1,466.58$ & $1,223.38$ \\
\hline Bajaj Auto Ltd. & 802.91 & 705.85 & 617.09 & 484.51 \\
\hline
\end{tabular}

All the three companies have consistent growth of BVPS between the years 2016 and 2019.

\subsection{Earnings Per Share (EPS)}

Earnings per share (EPS) is a company's net profit divided by the number of common shares it has outstanding. EPS indicates how much money a company makes for each share of its stock and is a widely used metric for corporate profits.

It is one of the many indicators used by investors in picking up stocks. Investors generally compare EPS with the share price of the stock to determine the value of earnings.

\begin{tabular}{l} 
EPS = Net Income \\
\cline { 2 - 6 } Teighted Average Outstanding Shares \\
Table 8 Earnings Per Share (Rs.) \\
\begin{tabular}{|l|c|c|c|c|}
\hline \multicolumn{1}{c}{ Company } & $\mathbf{2 0 1 9}$ & $\mathbf{2 0 1 8}$ & $\mathbf{2 0 1 7}$ & $\mathbf{2 0 1 6}$ \\
\hline Axis Bank & 19.61 & 1.86 & 16.54 & 35.12 \\
\hline Bajaj Finserve Ltd. & 202.30 & 166.50 & 142.10 & 117.10 \\
\hline Bajaj Auto Ltd. & 170.30 & 145.80 & 141.00 & 140.30 \\
\hline
\end{tabular}
\end{tabular}


The values mentioned above are taken from the annual reports of the three companies between the years 2016 and 2019. There is a consistent growth of EPS for Bajaj Finserve Limited and Bajaj Auto Limited. Whereas, the EPS of Axis Bank Limited has an oscillation between the years 2016 and 2019. This gives a positive sign for an investor to invest in Bajaj Finserve and Bajaj Auto.

\section{STOCK PRICE ANALYSIS IN CASH MARKET}

The daily closing stock prices of companies - Axis Bank, Bajaj Finserve and Bajaj Auto, listed at National Stock Exchange (NSE) were sourced for a period of 100 days (see Appendix A),i.e., from 4th May, 2020 to 13th August, 2020, and they are tabulated with price swings. For evaluating the volatility of the stocks, the coefficient of variation is found in each case to decide which stock is more volatile based on the 100-day data

Table 9 Showing Stock Prices and Price Swings of Axis Bank Limited (Rs.)

\begin{tabular}{|c|c|c|}
\hline Date & Stock Price & Price Swing \\
\hline $04-05-2020$ & 402.8 & - \\
$05-05-2020$ & 389 & -13.8 \\
$06-05-2020$ & 388.85 & -0.15 \\
$07-05-2020$ & 397.35 & 8.5 \\
$08-05-2020$ & 382.05 & -15.3 \\
$11-05-2020$ & 379.55 & -2.5 \\
$12-05-2020$ & 386.85 & 7.3 \\
$13-05-2020$ & 414 & 27.15 \\
$14-05-2020$ & 401.95 & -12.05 \\
$15-05-2020$ & 388.55 & -13.4 \\
$18-05-2020$ & 358.8 & -29.75 \\
$19-05-2020$ & 354.5 & -4.3 \\
$20-05-2020$ & 362.5 & 8 \\
$21-05-2020$ & 357.2 & -5.3 \\
$22-05-2020$ & 336.95 & -20.25 \\
$26-05-2020$ & 341.3 & 4.35 \\
$27-05-2020$ & 387 & 45.7 \\
$28-05-2020$ & 390.95 & 3.95 \\
$29-05-2020$ & 384.95 & -6 \\
$01-06-2020$ & 396.95 & 12 \\
$02-06-2020$ & 410.1 & 13.15 \\
$03-06-2020$ & 409.55 & -0.55 \\
$04-06-2020$ & 394.35 & -15.2 \\
$05-06-2020$ & 405.3 & 10.95 \\
$08-06-2020$ & 430.25 & 24.95 \\
$09-06-2020$ & 420.05 & -10.2 \\
$10-06-2020$ & 427.45 & 7.4 \\
$11-06-2020$ & 413.45 & -14 \\
$12-06-2020$ & 408 & -5.45 \\
$15-06-2020$ & 389.6 & -18.4 \\
$16-06-2020$ & 381.55 & -8.05 \\
$17-06-2020$ & 389.6 & 8.05 \\
$18-06-2020$ & 405.4 & 15.8 \\
$19-06-2020$ & 417.05 & 11.65 \\
$22-06-2020$ & 430.15 & 13.1 \\
$23-06-2020$ & 443.65 & 13.5 \\
$24-06-2020$ & 424.65 & -19 \\
\hline & & \\
\hline
\end{tabular}

\begin{tabular}{|c|c|c|}
\hline Date & Stock Price & Price Swing \\
\hline $25-06-2020$ & 421.7 & -2.95 \\
$26-06-2020$ & 424.85 & 3.15 \\
$29-06-2020$ & 404.8 & -20.05 \\
$30-06-2020$ & 406.65 & 1.85 \\
$01-07-2020$ & 433.25 & 26.6 \\
$02-07-2020$ & 423.2 & -10.05 \\
$03-07-2020$ & 428.45 & 5.25 \\
$06-07-2020$ & 434 & 5.55 \\
$07-07-2020$ & 447.35 & 13.35 \\
$08-07-2020$ & 444.15 & -3.2 \\
$09-07-2020$ & 453.75 & 9.6 \\
$10-07-2020$ & 439.6 & -14.15 \\
$13-07-2020$ & 440.05 & 0.45 \\
$14-07-2020$ & 417.7 & -22.35 \\
$15-07-2020$ & 426.65 & 8.95 \\
$16-07-2020$ & 434 & 7.35 \\
$17-07-2020$ & 433.1 & -0.9 \\
$20-07-2020$ & 434 & 0.9 \\
$21-07-2020$ & 446.2 & 12.2 \\
$22-07-2020$ & 478.95 & 32.75 \\
$23-07-2020$ & 460.85 & -18.1 \\
$24-07-2020$ & 445.6 & -15.25 \\
$27-07-2020$ & 431.55 & -14.05 \\
$28-07-2020$ & 437.05 & 5.5 \\
$29-07-2020$ & 440.9 & 3.85 \\
$30-07-2020$ & 425.85 & -15.05 \\
$31-07-2020$ & 431.65 & 5.8 \\
$03-08-2020$ & 417.35 & -14.3 \\
$04-08-2020$ & 429.15 & 11.8 \\
$05-08-2020$ & 434.95 & 5.8 \\
$06-08-2020$ & 433 \\
$07-08-2020$ & 433.3 & -1.95 \\
$10-08-2020$ & 431.1 & 0.3 \\
$11-08-2020$ & 448 & -2.2 \\
$12-08-2020$ & 451.1 & 16.9 \\
$13-08-2020$ & 448.1 & 3.1 \\
& & \\
\hline
\end{tabular}


Model for Identifying Volatility in Financial Markets with the help of NSE Listed Stocks with special reference to Axis Bank Limited, Bajaj Fin serve Limited and Bajaj Auto Limited during Post COVID19 in India-An Exploratory Study

Table 10 Showing Stock Prices and Price Swings of Bajaj Finserve Limited (Rs.)

\begin{tabular}{|c|c|c|}
\hline Date & Stock Price & Price Swing \\
\hline $04-05-2020$ & $4,762.35$ & - \\
$05-05-2020$ & $4,629.20$ & -133.15 \\
$06-05-2020$ & $4,692.90$ & 63.70 \\
$07-05-2020$ & $4,673.40$ & -19.50 \\
$08-05-2020$ & $4,601.00$ & -72.40 \\
$11-05-2020$ & $4,558.00$ & -43.00 \\
$12-05-2020$ & $4,668.20$ & 110.20 \\
$13-05-2020$ & $4,816.40$ & 148.20 \\
$14-05-2020$ & $4,790.25$ & -26.15 \\
$15-05-2020$ & $4,723.25$ & -67.00 \\
$18-05-2020$ & $4,465.45$ & -257.80 \\
$19-05-2020$ & $4,535.45$ & 70.00 \\
$20-05-2020$ & $4,702.05$ & 166.60 \\
$21-05-2020$ & $4,539.20$ & -162.85 \\
$22-05-2020$ & $4,315.55$ & -223.65 \\
$26-05-2020$ & $4,097.25$ & -218.30 \\
$27-05-2020$ & $4,258.05$ & 160.80 \\
$28-05-2020$ & $4,285.95$ & 27.90 \\
$29-05-2020$ & $4,404.90$ & 118.95 \\
$01-06-2020$ & $4,763.20$ & 358.30 \\
$02-06-2020$ & $5,184.70$ & 421.50 \\
$03-06-2020$ & $5,286.05$ & 101.35 \\
$04-06-2020$ & $5,136.80$ & -149.25 \\
$05-06-2020$ & $5,236.40$ & 99.60 \\
$08-06-2020$ & $5,440.95$ & 204.55 \\
$09-06-2020$ & $5,310.80$ & -130.15 \\
$10-06-2020$ & $5,223.00$ & -87.80 \\
$11-06-2020$ & $5,158.00$ & -65.00 \\
$12-06-2020$ & $5,285.95$ & 127.95 \\
$15-06-2020$ & $5,103.60$ & -182.35 \\
$16-06-2020$ & $5,128.30$ & 24.70 \\
$17-06-2020$ & $5,036.95$ & -91.35 \\
$18-06-2020$ & $5,427.55$ & 390.60 \\
$19-06-2020$ & $5,899.80$ & 472.25 \\
$22-06-2020$ & $6,183.55$ & 283.75 \\
$23-06-2020$ & $6,319.65$ & 136.10 \\
$24-06-2020$ & $6,046.05$ & -273.60 \\
\hline
\end{tabular}

\begin{tabular}{|c|c|c|}
\hline Date & Stock Price & Price Swing \\
\hline $25-06-2020$ & $6,021.15$ & -24.90 \\
$26-06-2020$ & $5,946.30$ & -74.85 \\
$29-06-2020$ & $5,890.20$ & -56.10 \\
$30-06-2020$ & $5,845.15$ & -45.05 \\
$01-07-2020$ & $6,169.35$ & 324.20 \\
$02-07-2020$ & $6,188.80$ & 19.45 \\
$03-07-2020$ & $6,149.40$ & -39.40 \\
$06-07-2020$ & $6,285.00$ & 135.60 \\
$07-07-2020$ & $6,589.60$ & 304.60 \\
$08-07-2020$ & $6,405.40$ & -184.20 \\
$09-07-2020$ & $6,569.20$ & 163.80 \\
$10-07-2020$ & $6,483.80$ & -85.40 \\
$13-07-2020$ & $6,461.45$ & -22.35 \\
$14-07-2020$ & $6,243.45$ & -218.00 \\
$15-07-2020$ & $6,168.45$ & -75.00 \\
$16-07-2020$ & $6,264.90$ & 96.45 \\
$17-07-2020$ & $6,361.60$ & 96.70 \\
$20-07-2020$ & $6,620.15$ & 258.55 \\
$21-07-2020$ & $6,384.35$ & -235.80 \\
$22-07-2020$ & $6,347.40$ & -36.95 \\
$23-07-2020$ & $6,381.35$ & 33.95 \\
$24-07-2020$ & $6,270.30$ & -111.05 \\
$27-07-2020$ & $6,173.20$ & -97.10 \\
$28-07-2020$ & $6,342.80$ & 169.60 \\
$29-07-2020$ & $6,328.60$ & -14.20 \\
$30-07-2020$ & $6,177.60$ & -151.00 \\
$31-07-2020$ & $6,205.90$ & 28.30 \\
$03-08-2020$ & $6,103.15$ & -102.75 \\
$04-08-2020$ & $6,162.00$ & 58.85 \\
$05-08-2020$ & $6,242.05$ & 80.05 \\
$06-08-2020$ & $6,295.70$ & 53.65 \\
$07-08-2020$ & $6,467.15$ & 171.45 \\
$10-08-2020$ & $6,402.80$ & -64.35 \\
$11-08-2020$ & $6,440.35$ & 37.55 \\
$12-08-2020$ & $6,357.20$ & -83.15 \\
$13-08-2020$ & $6,350.00$ & -7.20 \\
\hline
\end{tabular}


Table 11 Stock Prices and Price Swings of Bajaj Auto Limited (Rs.)

\begin{tabular}{|c|c|c|}
\hline Date & Stock Price & Price Swing \\
\hline $04-05-2020$ & $2,442.15$ & \\
$05-05-2020$ & $2,422.80$ & -19.35 \\
$06-05-2020$ & $2,471.85$ & 49.05 \\
$07-05-2020$ & $2,406.55$ & -65.30 \\
$08-05-2020$ & $2,419.05$ & 12.50 \\
$11-05-2020$ & $2,566.30$ & 147.25 \\
$12-05-2020$ & $2,628.85$ & 62.55 \\
$13-05-2020$ & $2,696.00$ & 67.15 \\
$14-05-2020$ & $2,702.65$ & 6.65 \\
$15-05-2020$ & $2,663.60$ & -39.05 \\
$18-05-2020$ & $2,477.45$ & -186.15 \\
$19-05-2020$ & $2,509.30$ & 31.85 \\
$20-05-2020$ & $2,557.75$ & 48.45 \\
$21-05-2020$ & $2,640.35$ & 82.60 \\
$22-05-2020$ & $2,553.75$ & -86.60 \\
$26-05-2020$ & $2,564.85$ & 11.10 \\
$27-05-2020$ & $2,580.55$ & 15.70 \\
$28-05-2020$ & $2,600.00$ & 19.45 \\
$29-05-2020$ & $2,710.50$ & 110.50 \\
$01-06-2020$ & $2,758.30$ & 47.80 \\
$02-06-2020$ & $2,796.10$ & 37.80 \\
$03-06-2020$ & $2,783.40$ & -12.70 \\
$04-06-2020$ & $2,817.25$ & 33.85 \\
$05-06-2020$ & $2,776.30$ & -40.95 \\
$08-06-2020$ & $2,801.05$ & 24.75 \\
$09-06-2020$ & $2,791.15$ & -9.90 \\
$10-06-2020$ & $2,717.20$ & -73.95 \\
$11-06-2020$ & $2,718.55$ & 1.35 \\
$12-06-2020$ & $2,782.15$ & 63.60 \\
$15-06-2020$ & $2,728.55$ & -53.60 \\
$16-06-2020$ & $2,718.45$ & -10.10 \\
$17-06-2020$ & $2,689.45$ & -29.00 \\
$18-06-2020$ & $2,678.35$ & -11.10 \\
$19-06-2020$ & $2,674.35$ & -4.00 \\
$22-06-2020$ & $2,855.75$ & 181.40 \\
$23-06-2020$ & $2,862.15$ & 6.40 \\
$24-06-2020$ & $2,817.25$ & -44.90 \\
\hline
\end{tabular}

\begin{tabular}{|c|c|c|}
\hline Date & Stock Price & Price Swing \\
\hline $25-06-2020$ & $2,820.45$ & 3.20 \\
$26-06-2020$ & $2,867.60$ & 47.15 \\
$29-06-2020$ & $2,859.50$ & -8.10 \\
$30-06-2020$ & $2,826.05$ & -33.45 \\
$01-07-2020$ & $2,842.05$ & 16.00 \\
$02-07-2020$ & $2,879.35$ & 37.30 \\
$03-07-2020$ & $2,932.40$ & 53.05 \\
$06-07-2020$ & $2,897.10$ & -35.30 \\
$07-07-2020$ & $2,847.15$ & -49.95 \\
$08-07-2020$ & $2,852.45$ & 5.30 \\
$09-07-2020$ & $2,882.00$ & 29.55 \\
$10-07-2020$ & $2,894.70$ & 12.70 \\
$13-07-2020$ & $2,900.00$ & 5.30 \\
$14-07-2020$ & $2,899.50$ & -0.50 \\
$15-07-2020$ & $2,942.05$ & 42.55 \\
$16-07-2020$ & $2,942.30$ & 0.25 \\
$17-07-2020$ & $2,995.00$ & 52.70 \\
$20-07-2020$ & $3,002.55$ & 7.55 \\
$21-07-2020$ & $3,004.30$ & 1.75 \\
$22-07-2020$ & $2,985.40$ & -18.90 \\
$23-07-2020$ & $3,032.10$ & 46.70 \\
$24-07-2020$ & $2,985.50$ & -46.60 \\
$27-07-2020$ & $3,020.50$ & 35.00 \\
$28-07-2020$ & $3,119.35$ & 98.85 \\
$29-07-2020$ & $3,099.30$ & -20.05 \\
$30-07-2020$ & $3,048.95$ & -50.35 \\
$31-07-2020$ & $3,004.95$ & -44.00 \\
$03-08-2020$ & $2,917.45$ & -87.50 \\
$04-08-2020$ & $2,969.95$ & 52.50 \\
$05-08-2020$ & $3,009.00$ & 39.05 \\
$06-08-2020$ & $2,991.80$ & -17.20 \\
$07-08-2020$ & $3,001.70$ & 9.90 \\
$10-08-2020$ & $2,993.80$ & -7.90 \\
$11-08-2020$ & $3,007.45$ & 13.65 \\
$12-08-2020$ & $3,023.00$ & 15.55 \\
$13-08-2020$ & $3,017.95$ & -5.05 \\
\hline
\end{tabular}

Table 12

\begin{tabular}{|l|c|c|c|}
\hline \multicolumn{1}{|c|}{ Price Swing (100days) } & Axis Bank Ltd. & Bajaj Finserve Ltd. & Bajaj Auto Ltd. \\
\hline No. of Upswings & 38 & 35 & 43 \\
\hline No. of Downswings & 34 & 37 & 29 \\
\hline
\end{tabular}

Table 13

\begin{tabular}{|l|l|}
\hline \multicolumn{2}{|c|}{ Axis Bank Limited } \\
\hline Average Price (Rs.) & 414.71 \\
\hline Standard Deviation & 28.76 \\
\hline Coefficient of Variation (CV) & 6.93 \\
\hline
\end{tabular}

Table 14

\begin{tabular}{|l|l|}
\hline \multicolumn{2}{|c|}{ Bajaj Finserve Limited } \\
\hline Average Price (Rs.) & $5,613.50$ \\
\hline Standard Deviation & 774.30 \\
\hline Coefficient of Variation (CV) & 13.79 \\
\hline
\end{tabular}


Model for Identifying Volatility in Financial Markets with the help of NSE Listed Stocks with special reference to Axis Bank Limited, Bajaj Fin serve Limited and Bajaj Auto Limited during Post COVID19 in India-An Exploratory Study

Table 15

\begin{tabular}{|l|l|}
\hline \multicolumn{2}{|c|}{ Bajaj Auto Limited } \\
\hline Average Price (Rs.) & $2,804.04$ \\
\hline Standard Deviation & 183.24 \\
\hline Coefficient of Variation (CV) & 6.53 \\
\hline
\end{tabular}

- In case of Axis Bank and Bajaj Auto, the number of upswings exceed the number of down- swings, which is a positive sign. Whereas, for Bajaj Finserve, the number of upswings fall behind the number of downswings by 2 .

- The CV of Bajaj Finserve stock prices is 13.79, which is more than the CV of Axis bank and Bajaj Auto stock prices. It clearly depicts that Bajaj Finserve stock is more volatile than that of Axis Bank and Bajaj Auto.

\section{FUTURE PRICE ANALYSIS IN DERIVATIVE MARKET}

The daily closing Future prices of Axis Bank, Bajaj Finserve and Bajaj Auto, listed at National Stock Exchange (NSE) were sourced for a period of last 90 days (see Appendix B). The price swings are determined and tabulated. For evaluating the volatility of the futures, the coefficient of variation is found in each case to decide which future is more volatile based on the 90-day data.

Table 16 Future Prices and Price Swings of Axis Bank Limited (Rs.)

\begin{tabular}{|c|c|c|}
\hline Date & Future Price & Price Swing \\
\hline 26-Jun-2020 & 426.65 & - \\
29-Jun-2020 & 405.25 & -21.4 \\
30-Jun-2020 & 404.2 & -1.05 \\
01-Jul-2020 & 436.8 & 32.6 \\
02-Jul-2020 & 424 & -12.8 \\
03-Jul-2020 & 429.6 & 5.6 \\
06-Jul-2020 & 436.3 & 6.7 \\
07-Jul-2020 & 449.9 & 13.6 \\
08-Jul-2020 & 445.65 & -4.25 \\
09-Jul-2020 & 455.65 & 10 \\
10-Jul-2020 & 444 & -11.65 \\
13-Jul-2020 & 444.3 & 0.3 \\
14-Jul-2020 & 421.4 & -22.9 \\
15-Jul-2020 & 429.2 & 7.8 \\
16-Jul-2020 & 437 & 7.8 \\
17-Jul-2020 & 436.9 & -0.1 \\
20-Jul-2020 & 435.2 & -1.7 \\
21-Jul-2020 & 450 & 14.8 \\
22-Jul-2020 & 481.95 & 31.95 \\
23-Jul-2020 & 464.6 & -17.35 \\
24-Jul-2020 & 448.85 & -15.75 \\
27-Jul-2020 & 434.7 & -14.15 \\
28-Jul-2020 & 440.45 & 5.75 \\
29-Jul-2020 & 443.55 & 3.1 \\
30-Jul-2020 & 427.9 & -15.65 \\
31-Jul-2020 & 434.9 & 7 \\
03-Aug-2020 & 420.3 & -14.6 \\
04-Aug-2020 & 431.15 & 10.85 \\
\hline
\end{tabular}

\begin{tabular}{|c|c|c|}
\hline Date & Future Price & Price Swing \\
\hline 05-Aug-2020 & 437.2 & 6.05 \\
06-Aug-2020 & 434.5 & -2.7 \\
07-Aug-2020 & 435.8 & 1.3 \\
10-Aug-2020 & 434.65 & -1.15 \\
11-Aug-2020 & 451.55 & 16.9 \\
12-Aug-2020 & 453.3 & 1.75 \\
13-Aug-2020 & 450.45 & -2.85 \\
14-Aug-2020 & 438.15 & -12.3 \\
17-Aug-2020 & 440 & 1.85 \\
18-Aug-2020 & 448.15 & 8.15 \\
19-Aug-2020 & 446.7 & -1.45 \\
20-Aug-2020 & 436.75 & -9.95 \\
21-Aug-2020 & 442.65 & 5.9 \\
24-Aug-2020 & 448.35 & 5.7 \\
25-Aug-2020 & 453.75 & 5.4 \\
26-Aug-2020 & 465.2 & 11.45 \\
27-Aug-2020 & 474.75 & 9.55 \\
28-Aug-2020 & 511.4 & 36.65 \\
31-Aug-2020 & 497.75 & -13.65 \\
01-Sep-2020 & 488.8 & -8.95 \\
02-Sep-2020 & 486.1 & -2.7 \\
03-Sep-2020 & 476.05 & -10.05 \\
04-Sep-2020 & 457.25 & -18.8 \\
07-Sep-2020 & 460.55 & 3.3 \\
08-Sep-2020 & 445.65 & -14.9 \\
09-Sep-2020 & 433.3 & -12.35 \\
10-Sep-2020 & 447.3 & 14 \\
11-Sep-2020 & 448.5 & 1.2 \\
\hline
\end{tabular}


Table 17 Future Prices and Price Swings of Bajaj Finserve Limited (Rs.)

\begin{tabular}{|c|c|c|}
\hline Date & Future Price & Price Swing \\
\hline 26-Jun-2020 & 6091.85 & \\
29-Jun-2020 & 5950 & -141.85 \\
30-Jun-2020 & 5900 & -50 \\
01-Jul-2020 & 6089 & 189 \\
02-Jul-2020 & 6089 & 0 \\
03-Jul-2020 & 6089 & 0 \\
06-Jul-2020 & 6089 & 0 \\
07-Jul-2020 & 6610.95 & 521.95 \\
08-Jul-2020 & 6425.3 & -185.65 \\
09-Jul-2020 & 6600 & 174.7 \\
10-Jul-2020 & 6572.95 & -27.05 \\
13-Jul-2020 & 6523 & -49.95 \\
14-Jul-2020 & 6320.05 & -202.95 \\
15-Jul-2020 & 6388.05 & 68 \\
16-Jul-2020 & 6388.05 & 0 \\
17-Jul-2020 & 6384.7 & -3.35 \\
20-Jul-2020 & 6474.9 & 90.2 \\
21-Jul-2020 & 6421.85 & -53.05 \\
22-Jul-2020 & 6300 & -121.85 \\
23-Jul-2020 & 6395 & 95 \\
24-Jul-2020 & 6280 & -115 \\
27-Jul-2020 & 6196.35 & -83.65 \\
28-Jul-2020 & 6395 & 198.65 \\
29-Jul-2020 & 6369.55 & -25.45 \\
30-Jul-2020 & 6213.1 & -156.45 \\
31-Jul-2020 & 6245.4 & 32.3 \\
03-Aug-2020 & 6145.25 & -100.15 \\
04-Aug-2020 & 6211 & 65.75 \\
\hline
\end{tabular}

\begin{tabular}{|c|c|c|}
\hline Date & Future Price & Price Swing \\
\hline 05-Aug-2020 & 6292.9 & 81.9 \\
06-Aug-2020 & 6341.55 & 48.65 \\
07-Aug-2020 & 6522.8 & 181.25 \\
10-Aug-2020 & 6444.5 & -78.3 \\
11-Aug-2020 & 6490.45 & 45.95 \\
12-Aug-2020 & 6402.5 & -87.95 \\
13-Aug-2020 & 6401.3 & -1.2 \\
14-Aug-2020 & 6281.35 & -119.95 \\
17-Aug-2020 & 6317.7 & 36.35 \\
18-Aug-2020 & 6381.75 & 64.05 \\
19-Aug-2020 & 6334.6 & -47.15 \\
20-Aug-2020 & 6315.9 & -18.7 \\
21-Aug-2020 & 6312.05 & -3.85 \\
24-Aug-2020 & 6438.25 & 126.2 \\
25-Aug-2020 & 6540.7 & 102.45 \\
26-Aug-2020 & 6598.2 & 57.5 \\
27-Aug-2020 & 6548.45 & -49.75 \\
28-Aug-2020 & 6575.4 & 26.95 \\
31-Aug-2020 & 6208.75 & -366.65 \\
01-Sep-2020 & 6387.45 & 178.7 \\
02-Sep-2020 & 6388.25 & 0.8 \\
03-Sep-2020 & 6386.65 & -1.6 \\
04-Sep-2020 & 6296.3 & -90.35 \\
07-Sep-2020 & 6271.15 & -25.15 \\
08-Sep-2020 & 6235.3 & -35.85 \\
09-Sep-2020 & 6058.5 & -176.8 \\
10-Sep-2020 & 6155.05 & 96.55 \\
11-Sep-2020 & 6153.35 & -1.7 \\
\hline
\end{tabular}

Table 18 Showing Future Prices and Price Swings of Bajaj Auto Limited (Rs.)

\begin{tabular}{|c|c|c|}
\hline Date & Future Price & Price Swing \\
\hline 26-Jun-2020 & 2853.55 & \\
29-Jun-2020 & 2853.55 & 0 \\
30-Jun-2020 & 2853.55 & 0 \\
01-Jul-2020 & 2853.55 & 0 \\
02-Jul-2020 & 2853.55 & 0 \\
03-Jul-2020 & 2853.55 & 0 \\
06-Jul-2020 & 2853.55 & 0 \\
07-Jul-2020 & 2853.55 & 0 \\
08-Jul-2020 & 2850 & -3.55 \\
09-Jul-2020 & 2878.45 & 28.45 \\
10-Jul-2020 & 2907.95 & 29.5 \\
13-Jul-2020 & 2907.95 & 0 \\
14-Jul-2020 & 2909.75 & 1.8 \\
15-Jul-2020 & 2909.75 & 0 \\
16-Jul-2020 & 2909.75 & 0 \\
17-Jul-2020 & 2909.75 & 0 \\
20-Jul-2020 & 2909.75 & 0 \\
21-Jul-2020 & 2909.75 & 0 \\
22-Jul-2020 & 2956 & 46.25 \\
23-Jul-2020 & 2956 & 0 \\
24-Jul-2020 & 2956 & 0 \\
27-Jul-2020 & 3003.65 & 47.65 \\
28-Jul-2020 & 3132.5 & 128.85 \\
29-Jul-2020 & 3113.5 & -19 \\
30-Jul-2020 & 3063.95 & -49.55 \\
31-Jul-2020 & 3020.15 & -43.8 \\
03-Aug-2020 & 2941.7 & -78.45 \\
04-Aug-2020 & 2989.5 & 47.8 \\
\hline
\end{tabular}

\begin{tabular}{|c|c|c|}
\hline Date & Future Price & Price Swing \\
\hline 05-Aug-20 & 3023.4 & 33.9 \\
06-Aug-20 & 3011.2 & -12.2 \\
07-Aug-20 & 3023.7 & 12.5 \\
10-Aug-20 & 3015.7 & -8 \\
11-Aug-20 & 3032.5 & 16.8 \\
12-Aug-20 & 3035.1 & 2.6 \\
13-Aug-20 & 3035.55 & 0.45 \\
14-Aug-20 & 3000 & -35.55 \\
17-Aug-20 & 3129.25 & 129.25 \\
18-Aug-20 & 3119.2 & -10.05 \\
19-Aug-20 & 3077.55 & -41.65 \\
20-Aug-20 & 3054.1 & -23.45 \\
21-Aug-20 & 3070.4 & 16.3 \\
24-Aug-20 & 3053.35 & -17.05 \\
25-Aug-20 & 3023.45 & -29.9 \\
26-Aug-20 & 3097.5 & 74.05 \\
27-Aug-20 & 3051.35 & -46.15 \\
28-Aug-20 & 3023.95 & -27.4 \\
31-Aug-20 & 2980.9 & -43.05 \\
01-Sep-20 & 2974.95 & -5.95 \\
02-Sep-20 & 2899.1 & -75.85 \\
03-Sep-20 & 2908.95 & 9.85 \\
04-Sep-20 & 2898.8 & -10.15 \\
07-Sep-20 & 2909.25 & 10.45 \\
08-Sep-20 & 2911.15 & 1.9 \\
09-Sep-20 & 2923.65 & 12.5 \\
10-Sep-20 & 2925.25 & 1.6 \\
11-Sep-20 & 2924.25 & -1 \\
\hline & & \\
\hline
\end{tabular}


Model for Identifying Volatility in Financial Markets with the help of NSE Listed Stocks with special reference to Axis Bank Limited, Bajaj Fin serve Limited and Bajaj Auto Limited during Post COVID19 in India-An Exploratory Study

Table 19

\begin{tabular}{|l|c|c|c|}
\hline \multicolumn{1}{|c|}{ Price Swing (100days) } & Axis Bank Ltd. & Bajaj Finserve Ltd. & Bajaj Auto Ltd. \\
\hline No. of Upswings & 29 & 22 & 20 \\
\hline No. of Downswings & 26 & 29 & 20 \\
\hline
\end{tabular}

Table 20

\begin{tabular}{|l|l|}
\hline \multicolumn{2}{|c|}{ Axis Bank Limited } \\
\hline Average Price (Rs.) & 446.16 \\
\hline Standard Deviation & 20.43 \\
\hline Coefficient of Variation (CV) & 4.58 \\
\hline
\end{tabular}

Table 21

\begin{tabular}{|l|l|}
\hline \multicolumn{2}{|c|}{ Bajaj Finserve Limited } \\
\hline Average Price (Rs.) & 6325.70 \\
\hline Standard Deviation & 166.10 \\
\hline Coefficient of Variation (CV) & 2.63 \\
\hline
\end{tabular}

Table 22

\begin{tabular}{|l|l|}
\hline \multicolumn{2}{|c|}{ Bajaj Auto Limited } \\
\hline Average Price (Rs.) & 2966.03 \\
\hline Standard Deviation & 83.48 \\
\hline Coefficient of Variation (CV) & 2.81 \\
\hline
\end{tabular}

- In case of Axis Bank and Bajaj Auto, the number of upswings exceed the number of down- swings, which is a positive sign. Whereas, for Bajaj Finserve, the number of upswings fall behind the number of downswings by 2 .

- The CV of Bajaj Finserve and Bajaj Auto Future prices are 2.63 and 2.81 respectively, which is better than the CV of Axis bank which stands at 4.58. From this, it can be concluded that Axis Bank Futures are more volatile than Bajaj Finserve and Bajaj Auto. Investing in the former would be more riskier than the latter.

\section{CONCLUSION}

Based on the analysis done from the collected data, in the equity segment, it would be better to invest in stocks of Axis Bank and Bajaj Auto instead of Bajaj Finserve. The stocks of Bajaj Finserve possesses double the risk of the other two. The EPS, BVPS and CFO show a positive sign towards investing in Bajaj Auto.

In the derivative segment, though Axis Bank has less number of downswings, it is more volatile than Bajaj Finserve and Bajaj Auto. So, It would be better to invest in the Bajaj group. The EPS, BVPS and CFO again show a positive sign towards investing in Bajaj Auto.

Also, while considering both the financial ratio analysis and the market price data of stocks and futures, it would be fine for an investor to invest his money in Bajaj Auto stocks or futures and gain less riskier returns when compared with Axis Bank and Bajaj Finserve. 


\section{REFERENCES}

[1] Axis Bank-Annual Reports https : //www.axisbank.com/shareholders corner/shareholdersinformation/annual reports

[2] Bajaj Finserve-Annual Reports https : //www.bajajfinserv.in/finserv - investor - relations annual - reports

[3] Bajaj Auto-Annual Reports https : //www.bajajauto.com/investors/annual - reports

[4] Financial Ratios and definitions https : //www.investopedia.com/

[5] Stock Prices for Axis Bank, Bajaj Finserve and Bajaj Auto https : //www.nseindia.com/get quotes/equity?symbol = AXISBANK https : //www.nseindia.com/get - quotes/equity? symbol $=$ BAJAJFINSV https : //www.nseindia.com/get - quotes/equity?symbol = BAJAJ - AUTO

[6] Future Prices for past 90 days www.nseindia.com/products/content/derivatives/equities/historical.htm

[7] www.moneycontrol.com

[8] Barua S K \& Varma J R (1991b), "Mastershares: A Bonanza for Large Investors", Vikalpa Vol. 16,No. 1 (Jan- Mar), p. 29-34. 3.

[9] Barua S K \& Varma J R (1991a), "Indian Convertible Bonds with Unspecified Terms: A Valuation Model", Working Paper No. 991, (Jul-Sep), Indian Institute of Management, Ahmedabad 